\title{
Política pública y educación indígena escolarizada en México
}

\author{
Héctor Muñoz Cruz*
}

\begin{abstract}
RESUMO: La educación de los alumnos indígenas, en cualesquiera de sus modalidades, todavía no tiene el status de cuestión principal en la sociedad mexicana actual, a pesar de haber ganado gran presencia en la actividad política y en los escenarios culturales. Aunque el debate político haga patente la incapacidad para conectar el desarrollo educativo con las reformas políticas y económicas, que hoy dividen profundamente a la nación mexicana, todavía no se visualizan los cambios estructurales correspondientes a un Estado que asume coherentemente su etnodiversidad.
\end{abstract}

Desde el sistema educacional, las demandas sobre educación bilíngüe y multiculturalidad han resultado mucho menos influyentes que la doctrina de la modernización de la educación básica y menos interesantes que la pugna por la hegemonía de ciertos paradigmas pedagógicos en las cúpulas de las instituciones educacionales. En suma, las comunidades y escuelas indomexicanas deberán reconquistar su educación, al mismo tiempo que logran participación y capacidad de decisión frente a las instituciones gubernamentales.

Palavras-chave: Educação bilingüe-intercultural, políticas de educação indígena, educação oficial, educação indo-americana, povos indígenas do México

\footnotetext{
* Professor-pesquisador da Universidade Autônoma Metropolitana, México-DF.

E-mail: hmunoz2@prodigy.net.mx
} 
Cada cual en este mundo cuenta el cuento a su manera y lo hace ver de otro modo en la mente de cualquiera...

(Curet Alonso, La tirana)

\section{Introducción}

La educación indígena escolarizada en México muestra las características de un proceso de largo aliento que se constituye, se detiene o se reconstituye, según los casos, en estrecha concatenación con la reforma política del Estado y los cambios socioeconómicos inspirados en concepciones neoliberales del desarrollo nacional.

En la última década, ha conquistado una dimensión política e institucional de primer orden y ha vuelto mucho más visibles a los pueblos indomexicanos. También ha acelerado la creación de nuevas bases jurídicas y políticas públicas de educación escolarizada, cambiando paulatinamente un adverso proceso en la educación pública. Sin embargo, con todo ese inmenso potencial de cambio y enriquecimiento ético, ese proceso parece debilitarse y perder presencia en la sociedad mexicana.

Un resultado ideológico significativo de ese cambio es la sustitución irreversible del paradigma de la educación bilingüe bicultural por la llamada propuesta intercultural bilingüe, hasta el momento. Antes, en los años 70 , la doctrina bipolar del bilingüismo y biculturalismo en la educación indígena había reemplazado la vieja propuesta integracionista de los años 30 llamada "educación bilingüe".

\section{Cuadro 1}

Paradigmas de la educación indígena escolarizada en América Latina

\begin{tabular}{|c|c|}
\hline Educación bilingüe (EB) & Modelo democratizador \\
\hline Educación bilingüe bicultural (EBB) & $\begin{array}{l}\text { Modelo de capital humano y } \\
\text { de superación de la marginalidad }\end{array}$ \\
\hline $\begin{array}{l}\text { Educación bilingüe intercultural (EBI) } \\
\text { Educación intercultural bilingüe (EIB) } \\
\text { Educación indígena } \\
\text { Educación multicultural } \\
\text { Educación endógena } \\
\text { Educación propia } \\
\text { Etnoeducación }\end{array}$ & $\begin{array}{l}\text { Modelo liberal de capital humano } \\
\text { Modelo liberal de capital humano } \\
\text { Modelos críticos de resistencia }\end{array}$ \\
\hline
\end{tabular}


Es un hecho que han cambiado las concepciones sobre la educación y la escuela en las regiones indígenas, pero no de modo tan gradual y acumulativo como sugiere la secuencia anotada en el Cuadro 1 (supra). También es un hecho que la historia científica de esta lucha entre paradigmas de la educación indígena escolarizada todavía es una tarea por hacerse. La interacción actual entre el Estado mexicano y organizaciones indígenas permite la convergencia de modelos educativos de corte liberal con propuestas críticas de resistencia y emancipación que son incompatibles y que revelan los profundos desacuerdos en torno a esa cuestión. Así, problemas fundamentales de la educación indígena subsisten y permanecen sin solución, a pesar de los cambios paradigmáticos. Me refiero a problemas tales como las metodologías de enseñanza de la lengua materna y segunda lengua, la construcción étnica de la escuela bilingüe, el diseño de los contenidos curriculares, el fomento de la autoestima positiva e identidad étnica y la revitalización de las lenguas minorizadas. Sin embargo, una cosa podemos postular como segura: las diversas orientaciones curriculares reflejan la voluntad política de influir en el futuro de la sociedad y la identidad indígenas, redefiniendo el papel de la educación en el vasto esfuerzo de permitir la realización al mayor número de individuos de origen indo y afroamericano de los actuales países latinoamericanos (cf. Le Bot 1997, Gobierno Federal y EZLN 1996, Lesourne 1993). Sin embargo, la mayoría de las experiencias orientadas por el enfoque de la interculturalidad todavía no logran el arraigo y la cobertura escolar necesarias. Eso nos enseña que para poner en marcha una educación con esas características se requiere de esfuerzos muy diferentes a los aplicados hasta la fecha.

Pero, sin lugar a dudas, lo que destaca en el panorama continental, que incluye por cierto a México, son las adecuaciones que realizan los gobiernos nacionales por insertar la interculturalidad, la competitividad, la diversidad y la pluralidad democrática en algunas políticas públicas. Sobre la base de convergencias globales, cada país ha identificado y construido sus términos específicos de oferta de educación indígena, produciéndose un notable enriquecimiento y diversificación de las reformas educativas concernientes a los pueblos indígenas. Nicaragua, por ejemplo, debe resolver los problemas de educación indígena en relación con la autonomía y desarrollo socioeconómico de la costa caribe. Guatemala hace otro tanto en el contexto de los acuerdos de paz y de desarrollo sostenible, aprovechando la presencia y los recursos de agencias de cooperación. Chile se propuso en 1996 el objetivo de, en el plazo de tres años, contar con un diseño y una propuesta curricular y pedagógica de EIB, especialmente para los primeros 
años de formación de niños indígenas (Min. de Educ. de Chile 1996, p. 11). Colombia debate sobre la etnoeducación en conexión con el reconocimiento jurídico constitucional de la territorialidad de sus pueblos indígenas. Por su parte, el Ministerio de Educación brasileño plantea que

para las sociedades indígenas contemporáneas, la escuela es una institución originaria de otras formaciones sociales, que debe ser creativamente incorporada. Su readecuación implica normas propias en relación a los diversos aspectos curriculares. La organización curricular de la escuela indígena es un modo de concretizar el principio del respeto "a los procesos propios de aprendizaje" y al "pluralismo de ideas y concepciones pedagógicas". (MEC 1997, p. 37)

Bolivia se encuentra en el difícil proceso de validar la educación intercultural como una política de todo el sistema nacional, y con la ayuda de las agencias internacionales intenta cambios radicales en la formación y actualización de sus recursos docentes. En México, la flexibilidad curricular y los programas compensatorios se establecen como las principales estrategias del más reciente proyecto educativo. Entonces cabe preguntar ¿cuáles son los conceptos propios de la educación indígena del presente? Y sobre todo ¿qué estrategias emplear para desencadenar las transformaciones necesarias? En suma, la mayoría de los países latinoamericanos establecen nuevos proyectos educativos, los cuales sustancializan el concepto de desarrollo humano y profundizan la doctrina de la modernización educativa, con componentes de equidad, calidad, sustentabilidad y de productividad, en diversos sectores de la vida nacional.

En resumen, en México, en la condición de política pública, la educación indígena ha sido integrada a la reforma educativa nacional y otras reformas del Estado, para dar más poder a los actores locales, quienes todavía no perciben grandes beneficios en las reformas. Además, el enfoque intercultural bilingüe ha surgido con un fuerte posesionamiento en torno a la educación básica, en el sentido de acrecentar la calidad y extenderla a los grupos sociales que aún la reciben en forma insuficiente. Se ha introducido el postulado de bidireccionalidad de la acción educativa del Estado hacia los pueblos indios, a fin de combatir las formas manifiestas y encubiertas de racismo y promover una justa valoración de la contribución de los pueblos indios a la construcción histórica de la nación (idem, pp. 75-76). Por último, se intenta enriquecer el concepto de territorialidad, reconociendo la autodeterminación y soberanía de las comunidades indígenas. 
El panorama planteado implica modificar las concepciones sobre cómo opera y se arraiga un cambio en la educación. Hasta el momento, se han usado principalmente argumentaciones demográficas y las descripciones psicológicas, lingüísticas y sociolingüísticas de contextos indígenas para enfatizar la necesidad de cambios, pero de esas descripciones no se derivan los posibles caminos por seguir. Lo que se requiere es una conceptualización que resalte la unidad orgánica del sector educativo y enriquezca las interrelaciones entre las diferentes dimensiones del cambio integral de la educación indígena y, en ese marco, establezca las prioridades y las fases por cumplir. Para este efecto, propongo un modeb de desarrollo educativo en regiones indígenas, lo que implica la convergencia de cuatro niveles de acciones educativas, los cuales operan bajo determinaciones y formas de legitimidad provenientes de actores e instituciones diferentes. A continuación, comentaré casos de cada una de las dimensiones sugeridas.

\section{Cuadro 2}

Factores del desarrollo educativo en regiones indígenas

\begin{tabular}{|l|l|}
\hline Niveles & Conceptos \\
\hline $\begin{array}{l}1^{\underline{0}} \text { Políticas educativas, } \\
\text { proyectos de los sectores } \\
\text { sociales, debates } \\
\text { internacionales }\end{array}$ & $\begin{array}{l}\text { Discusiones y consensos políticos y segmentos de modelos } \\
\text { teóricos en torno a los objetivos y categorías para definir la } \\
\text { naturaleza social y papel de la educación en el desarrollo de la } \\
\text { sociedad indígena }\end{array}$ \\
\hline $2^{\underline{0} \text { Sistema educacional }}$ & $\begin{array}{l}\text { Organización y estrategias del gobierno para ejecutar las } \\
\text { doctrinas educativas oficiales }\end{array}$ \\
\hline $\begin{array}{l}3^{\underline{0}} \text { Modelos académicos o } \\
\text { de formación de la } \\
\text { educación indígena }\end{array}$ & $\begin{array}{l}\text { Diseños curriculares, propuestas metodológicas, técnicas } \\
\text { didácticas, constitución de la enseñanza y del aprendizaje } \\
\text { conforme a las características psicolingüísticas y } \\
\text { socioculturales del alumno indígena. }\end{array}$ \\
\hline $4^{\underline{0}}$ Prácticas escolares & $\begin{array}{l}\text { Acciones e ideologías educativas de maestros, padres y } \\
\text { autoridades comunitarias que constituyen las diferentes } \\
\text { realidades escolares }\end{array}$ \\
\hline
\end{tabular}

Desde esa óptica integral o sistémica, surgen principios y condiciones ideológicas para promover un gran cambio educativo que se 
oriente a establecer nuevos términos con el desarrollo económico y político en que "la democracia de nuestros países encontrará en la educación intercultural y bilingüe uno de los principales instrumentos para su consolidación" (Cárdenas 1997, p. 29). Sin embargo, el cambio educativo en una sociedad precisa revertir el fracaso institucional, el fracaso financiero y el fracaso científico de la escuela (Bayet, apud Lesourne 1993, p. 12), mediante un laborioso y frágil proceso de aperturas y negociaciones políticas, un amplísimo contexto de participación, muchas investigaciones diagnósticas, otra oferta coherente de formación docente, nuevos materiales didácticos, nuevas propuestas curriculares y estrategias eficientes para desencadenar el cambio. Siguiendo a Garmendia (1997), se trata de un proceso de legitimaciones, de progresividad, de participación y de cooperación entre los diferentes sectores de la sociedad nacional latinoamericana.

A modo de hipótesis, sugiero que la transformación o apropiación del enfoque intercultural bilingüe en regiones indígenas mexicanas provendrá principalmente del nivel de las realidades y prácticas escolares, en la medida que se democratice la gestión de las políticas de educación pública, mediante formas de control comunitario. El sistema educacional sólo reflejará las condiciones políticas imperantes. El nivel de los modelos académicos o de formación permanecerá invisible, hasta que se restablezcan las condiciones profesionales y las conexiones con la investigación de la educación indígena.

\section{Ideologías, políticas y proyectos educativos}

En la definición de las políticas educativas en regiones indígenas mexicanas, organizaciones comunitarias, agencias internacionales y funcionarios de la Secretaría de Educación Pública, por intermedio de la Dirección General de Educación Indígena, o investigadores de la educación intercultural bilingüe discrepan a menudo, a propósito del peso específico que se le debe asignar a la etnodiversidad, a los bilingüismos, a la interculturalidad contemporánea, a la formación docente y propugnan medidas muy diversas. Es decir, los distintos sectores sociales tienen grandes discrepancias en la definición de las políticas educativas y en los factores sobre los cuales sustentar la eficiencia y calidad de los aprendizajes y el desarrollo socioeducativo. Pero es un hecho positivo el 
que los debates se sitúen cada vez más en la perspectiva de visualizar beneficios concretos de un sistema escolar puesto en operación, lo cual nos aproxima lentamente a concreciones hacia una justicia social y educativa en favor de los sectores indígenas.

Las reformas "alternativas" que asignan carácter constitucional a los pueblos indios y facultades y facultades de autodeterminación para decidir sobre sus diversos proyectos provienen casi exclusivamente de organizaciones indígenas. Pero éstas no habrían logrado la atención, si no hubieran manifestado síntomas de la crisis política del Estado Mexicano. En efecto, entre 1991 y 1994 ocurren dos coyunturas que han transformado significativamente la situación política de los grupos étnicos indomexicanos.

En 1991, se proclamó oficialmente la descentralización del sistema educativo nacional y se decretaron reformas jurídicas que legislan sobre el derecho a la justicia, a la administración y a la educación, de acuerdo a las características culturales y linguísticas de los pueblos indoamericanos.

Es una situación conocida en México que los cambios institucionales giran en torno al problema de la legitimidad. Por una parte, los cambios sociopolíticos surgen como iniciativas o concesiones gubernamentales y, por otra, se plantean como logros de los movimientos sociales.

Una buena consecuencia de la lucha por tornar más visible la educación intercultural bilingüe es que ella ha producido una desconocida mayor participación de las comunidades, dando forma a nuevas organizaciones e instituciones para definir y operar la justicia, el gobierno y la educación. En educación, la participación de varios miles de nuevos agentes educativos significa una aproximación popular a la EIB, que no se había dado antes, en razón de las arraigadas prácticas verticalistas de las instituciones gubernamentales, y comienzan a discutirse de nuevo, diríamos a construirse, los cimientos de la política educativa. No sólo han proliferado los comités técnico-pedagógicos por idioma y por región étnica, sino que algunas organizaciones comunitarias indígenas inician gestiones de educación pública bilingüe e invierten esfuerzos y recursos financieros propios para apoyar el desarrollo educativo de sus hijos. No es seguro que haya una continuidad de los planes técnico-pedagógicos, pero quizás la cuestión académica no importe tanto ahora. 
El $1^{\circ}$ de enero de 1994, el levantamiento armado del EZLN en Chiapas desencadena el más reciente proceso de debate y lucha política en torno a la importancia de las culturas indígenas en el futuro del país, el cual ha generado negociaciones y grandes foros nacionales de consulta que han focalizado las cuestiones de participación y de cambio político y económico, relegando los espacios culturales y educativos a diversos grupos de intelectuales indígenas y no indígenas, que no han podido generar todavía una propuesta alternativa en materia de educación indígena.

Entre estas dos coyunturas aparece, por presión o iniciativa del Banco Mundial y del BID, una estrategia de transición que consiste en programas compensatorios en las áreas de mayor rezago educativo, estrategia que tiene a generalizarse en el continente. En lo que respecta al sistema nacional de educación indígena se establecieron estos programas en los Estados de mayor rezago socioeducativo. Se trata de una estrategia de cambios parciales, a fin de consolidar una eficiencia sobre mínimos educativos y a la vez de transferir lentamente la conducción de la gestión educativa a los gobiernos regionales, no a las comunidades. Existe la esperanza de que los beneficios de estos programas compensatorios se multipliquen en las demás áreas. Aunque no hay información suficiente, no es seguro que esto cause un cambio sustancial en el sistema de educación indígena, ya que las condiciones "excepcionales" no se darán en el conjunto de las escuelas.

\section{Política educativa 1995-2000}

El más reciente programa educativo del gobierno de México se enmarca en el concepto de desarrollo humano, que es una ampliación de la doctrina de la modernización educativa y que tiene componentes de equidad, sustentabilidad y de productividad. Dicha noción resulta inclasificable desde el punto de vista teórico y político. Su argumentación procede por referencias a gestiones destacadas de gobiernos mexicanos anteriores. Así, se asume una influencia de la educación popular y la acción de los maestros misioneros, propuesta de José Vasconcelos (1914; 1921-24). También reconoce la doctrina del impacto de la educación (Iibro de texto gratuito e igualdad de oportunidades) en la unidad nacional desarrollada por Jaime Torres Bodet (1952 y 1964-70). Igual influencia se reconoce en las ideas de Justo Sierra en torno a los principios de libertad de cátedra y de investigación (1905-1911). También se suscriben 
a modo de compromisos el mejoramiento de la preparación de los maestros y un compromiso singular con la población indígena y con la diversidad étnica y cultural del país que ha dado origen a la educación bilingüe y bicultural (SEP 1996, p. 10).

Los propósitos fundamentales que animan al programa de desarrollo educativo son la equidad, la calidad y la pertinencia de la educación (SEP 1996, pp. 12-13). Una de las ofertas principales de ese programa institucional es la educación básica, que se define en los siguientes términos:

En los linderos del fin de siglo conserva plena vigencia la necesidad de mejorar la calidad de la educación básica y de extenderla a los grupos sociales que aún la reciben en forma insuficiente. En la educación básica han de adquirirse valores esenciales, conocimientos fundamentales y competencias intelectuales que permitan aprender permanentemente; en ella se despiertan la curiosidad y el gusto por el saber y se forman hábitos de trabajos individuales y de grupo. El valor de una buena educación básica habrá de reflejarse en la calidad de vida personal y comunitaria, en la capacidad de adquirir destrezas para la actividad productiva y en el aprovechamiento pleno de las oportunidades de estudio en los niveles medio superior y superior. (p. 19)

Con respecto a los grupos indígenas, el programa oficial admite que:

La mayor parte de los grupos indígenas de México vive en condiciones de pobreza y marginación. Conforme a las cifras del censo de 1990, los grupos étnicos representan el $7 \%$ de la población nacional, aunque concentran a 26 por ciento de los analfabetos del país. A las dificultades ya señaladas de acceso e insuficiente infraestructura para llevar servicios educativos a estas regiones, se añade el monolingüismo indígena, que obliga a combinar el uso del español con las lenguas predominantes en cada región. La enseñanza en lenguas indígenas implica retos que apenas en los últimos lustros se han abordado. Todavía no se ha logrado una educación pertinente a estos grupos que ofrezca, simultáneamente, una formación que les facilite la integración a la vida nacional y les resulte más asequible y relevante en relación con su entorno. (SEP 1996, p. 75)

El nuevo programa educativo mexicano sigue considerando la diversidad lingüística como un problema central y complejo. Con base 
en el censo de 1990 reconoce, por una parte, una demanda educativa potencial de 1,2 millon de personas, hablantes de alguna de las más de 50 lenguas indígenas del país, sin que pueda establecerse con aceptable aproximación cuántos son monolingües y cuántos poseen algún grado apreciable de bilingüismo. Por otra, admite las enormes fallas del servicio resultantes de la discontinuidad lingüística entre escuela y comunidad y asume la necesidad de personal y de recursos didácticos que respondan a esa condición y proporcionen un servicio adecuado (p. 78).

Una novedad del programa es el postulado de bidireccionalidad de la acción educativa del Estado hacia los pueblos indios. A modo de dirección principal declara que la educación destinada específicamente a los grupos indígenas se adaptará a sus necesidades, demandas y condiciones de cultura y lengua, poblamiento, organización social y formas de producción y trabajo. La segunda se refiere a la población no indígena, a fin de combatir las formas manifiestas y encubiertas de racismo y promover una justa valoración de la contribución de los pueblos indios a la construcción histórica de la nación, el conocimiento de su situación y sus problemas y el reconocimiento de los aportes que realizan en todos los ámbitos de la vida del país (SEP 1996, pp. 75-76).

Otra novedad es la flexibilización del concepto de territorialidad, puesto que admite la inequidad sobre los migrantes de las zonas rurales hacia las ciudades de grupos de familias de diversas etnias que demandan servicios de educación básica. "El hecho de que los servicios para indígenas se hayan concentrado en las regiones de origen (...) hace prácticamente inexistente la experiencia institucional de ese tipo de servicios educativos en zonas urbanas" (SEP 1996, p. 79). En la actualidad sucede algo semejante con los programas binacionales en educación de adultos.

Pablo Latapí (1997, pp. 42-43), en sus conocidos artículos en la revista Proceso, considera que

el nuevo programa (en la parte relativa a la enseñanza básica) me suscita tres reflexiones. En primer lugar, (...) me parece advertir una creciente unidad orgánica en la política del sector (...). En segundo lugar, se advierte en el programa una concepción clara de la manera como se pretende fomentar la calidad educativa (...). 
Una tercera reflexión versa sobre las prioridades para el presente año, entre las cuales la educación indígena.

Desde la investigación

Otro aspecto interviniente en la formación de las políticas y proyectos educativos son los estudios científicos sobre las sociedades indomexicanas. Como en otros tantos campos de la realidad mexicana, la historia, las teorías y las prácticas de la educación indígena están estrechamente ligadas a discusiones e investigaciones de la antropología social. Pensadores tales como Julio de la Fuente, Gonzalo Aguirre Beltrán, Mauricio Swadesh, Guillermo Bonfil, Rodolfo Stavenhagen y Salomón Nahmad han producido importantes contribuciones para comprender la situación multiétnica y pluricultural del país, las cuales han sido apropiadas por las instituciones gubernamentales, dando forma a una avanzada política sociocultural del Estado mexicano, pero que no se ha traducido en una congruente política de gobierno ni en la necesaria comprensión y aceptación por parte de maestros y comunidades indígenas.

El "descubrimiento", análisis y conciencia inicial de la diversidad linguística y cultural de México es atribuible tanto a las coyunturas políticas como a la acumulación investigativa. Para los ciudadanos del México intercultural, ese descubrimiento duele, sorprende y fascina, aunque todavía no puede ser colocado en el desarrollo y en el futuro del país. Por ahora, sirve como sustento natural para cualquier modelo de educación indígena. Tan solo en los campos de la sociolingüística y antropología lingüística han ocurrido discusiones que repercuten o pueden repercutir en los sectores involucrados con la educación indígena. De un modo no exhaustivo, mencionamos algunas principales.

Estos ejemplos de propuestas y resultados sirven para mostrar la riqueza del conocimiento acumulado en las diversas ciencias sobre las culturas indomexicanas. Eso hace surgir la interrogante sobre el tipo de relaciones de la ciencia en México con los diversos niveles del desarrollo educativo. ¿Logran permear los discursos y prácticas de los sectores que construyen la política y de los que componen el sistema educacional? Talvez sean las instituciones formadoras de maestros bilingües y de especialistas las que más aprovechan las 
contribuciones de las investigaciones científicas, aunque de una manera todavía no metódica ni exhaustiva.

\title{
El sistema escolar de educación indígena
}

Cuatro son los principales retos del sistema educacional para alumnos indígenas: descentralización, participación o control comunitario de la educación, organización pedagógica y la formación y capacitación docente. Transcurridas las dos últimas décadas, cada uno de estos dominios empezaron teniendo dinámicas locales y han terminado por tomar el cauce de una estrategia gubernamental nacional y centralizada, que ha restablecido la capacidad de control de las instituciones gubernamentales. En esta ocasión, sólo nos referiremos al último.

En el continente existen dos experiencias ejemplares y polares sobre formación de maestros indígenas: el Programa de lquitos, Perú y el Sistema nacional formador de México. Por la importancia estratégica del tema en la actualidad latinoamericana, nos detendremos a comentar brevemente ambas experiencias.

\section{La experiencia de Iquitos, Perú}

\begin{abstract}
Uno de los aspectos más innovadores del Programa de Formación de Maestros Bilingües de la Amazonía peruana ha sido su marco institucional. En efecto, éste ha sido planteado en términos de coejecución entre una confederación indígena (Aidesep) y un instituto estatal de formación magisterial. Sus aportes más significativos, tanto a nivel de la formulación del proyecto sociopolítico que lo orienta, como lo que atañe a los aspectos de su organización y funcionamiento, derivan precisamente de este marco inédito en el país y el continente. (ISP Loreto 1997, p. 10)
\end{abstract}

Los maestros formados por el programa son concebidos como actores sociales que participarán en la profundización y difusión de los alcances y consecuencias de los derechos de los pueblos indígenas, entre los cuales son fundamentales los de autodeterminación y propiedad 
territorial (p. 17). Con ese fin, se postula un marco teórico para entender la realidad actual de los pueblos amazónicos con base en la comprensión del funcionamiento de las sociedades. En efecto, el programa asume que los pueblos indígenas que hoy en día viven en la Amazonía peruana provienen de sociedades que son variantes de una formación social económica, denominada tribal amazónica.

El enfoque de educación intercultural asumido por el programa se inscribe más bien en una perspectiva sociopolítica, a diferencia de otras propuestas que subrayan el aspecto cultural. Esa perspectiva se trabaja en dos niveles: de las relaciones entre pueblos indígenas y sociedad nacional; y de las relaciones que se establecen al interior de cada pueblo y que se expresan por el parentesco y por diversos mecanismos de intercambio. La cultura, entendida como el resultado de las relaciones de producción y comunicación que se establecen al interior de las diferentes unidades residenciales y entre ellas, y que además involucran a los diferentes miembros del universo socionatural indígena, se explica desde esa perspectiva global (p. 19).

Dadas las condiciones de dominación ideológica que han caracterizado la relación entre pueblos indígenas y sociedad nacional, ese programa propicia espacios tendientes a afirmar la cultura indígena, para que los estudiantes manifiesten sus contradicciones y prejuicios en relación a las concepciones y prácticas indígenas, y expresen sus temores y ambigüedades en relación a las propuestas que se van creando en el marco del programa. Dichas propuestas requieren respuestas creativas y críticas a las diversas y sutiles formas que presenta la dominación en la vida cotidiana (p. 19).

\section{El sistema formador de la Universidad Pedagógica Nacional}

México posee la mayor oferta formadora de maestros bilingües del continente. La Universidad Pedagógica Nacional ofrece una licenciatura de cuatro años en educación preescolar y primaria para maestros de escuelas indígenas, a través de una modalidad escolarizada (en la unidad central de la Ciudad de México) y de una modalidad semiescolarizada intensiva (en 74 unidades regionales de ese sistema universitario), atendiendo a unos 13 mil maestros bilingües en todo el país. Dicho programa fue creado en 1990 y se encuentra en estos momentos en una fase de evaluación. 
La magnitud de ese esfuerzo no está, ciertamente, exenta de problemas. En efecto, se pueden señalar tres aspectos problemáticos: primero, la formación de maestros en servicio activo ha orillado a una operación con una modalidad semiescolarizada, mediante la cual los maestros asisten sábados y domingos, quincenal o semanalmente, ya que no es posible comisionar a todos para que ingresen a una modalidad escolarizada, como el plantel de la Ciudad de México.

El modelo de formación en cuestión supone condiciones materiales para el desarrollo de un autodidactismo de los maestros-estudiantes y, significativamente, supone la adopción de estrategias cognitivas y de estudio, típicas de las instituciones universitarias tradicionales. Razones culturales asociadas a entornos con escasa funcionalidad de la lengua escrita en los respectivos contextos indígenas podrían explicar la adopción muy lenta de las estrategias cognitivas y de estudio previstas, de modo tal que estos maestros asisten a sus aulas universitarias sin el necesario proceso autodidacta de estudio y limitan el alcance y la calidad del trabajo en el aula.

En segundo término, la posibilidad de producir un impacto de cambio y mejoramiento de la enseñanza-aprendizaje en las escuelas indígenas se relaciona con la adecuada articulación entre la formación teórica y la práctica socioeducativa. El fenómeno que llaman "empirismo" en Nicaragua suele desarrollar en los maestros una relación no madura, de extrañamiento respecto de las fuentes técnicas y reflexiones conceptuales y, en ocasiones, una sacralización de la práctica. Los mismos programas consienten con ese empirismo y sitúan el análisis de la práctica docente como el foco integrador de la formación, sin ofrecer recursos de sistematización, provocando una gran corriente de descriptivismo anecdótico de los procesos escolares.

En tercer lugar, cabe mencionar la no menos problemática relación entre investigación educativa y docencia, que no comentaremos mayormente. La evaluación actualmente en curso en todo el país analiza éstas, entre otras cuestiones.

Otra enseñanza de esa importante experiencia es la formación de los formadores de maestros bilingües. Esa profesionalización o especialización en educación bilingüe ha implicado la captación de una gran cantidad de académicos que han debido enfrentarse a una experiencia formadora inédita y los avances son verdaderamente importantes, pero no suficientes. También en ese nivel se presenta la necesidad de la profesionalización mediante programas de posgrado. En este momento, 
existe una maestría en educación bilingüe en la Unidad Ajusco, Ciudad de México, en la Universidad Pedagógica, una maestría en linguística indoamericana en el Centro de Investigaciones y Estudios Superiores en Antropología Social, con el apoyo del Instituto Nacional Indigenista y una maestría en sociolinguística de la educación bilingüe y básica en la Unidad Oaxaca de la Universidad Pedagógica Nacional. Además, la Universidad Veracruzana de Xalapa ofrece a partir de este año una maestría en educación básica y lenguaje. También las unidades de Uruapan, Zamora, Zitacuáro y Morelia de la Universidad Pedagógica Nacional en el estado de Michoacán ofrecen una maestría en educación bilingüe.

En 1998 cobra forma el proyecto de Escuela Normal Bilingüe de Oaxaca, que adopta la concepción de la educación bilingüe e intercultural, con el propósito de preparar profesores indígenas biblingües que deberán atender los servicios del nivel inicial, preescolar y primaria, "a partir del conocimiento sistemático de su cultura y la promoción de los conocimientos regionales, nacionales y universales para el desarrollo integral de los pueblos indígenas" (leepo 1998, p. 2).

En la parte de fundamentación del documento programático, se afirma que

en la actualidad, a los indígenas no nos incomoda la idea de integrarnos a la dinámica del proceso de modernización, porque es evidente que los Pueblos Indígenas, en la medida que logren satisfacer sus necesidades elementales de carácter colectivo e individual, tomarán conciencia de su pertenencia a una colectividad más amplia (que es la sociedad nacional) y en una política de integración, participación e inclusión con enfoque acorde a sus expectativas, y asumirán su papel de mexicanos como cualquier otro individuo de la sociedad no indígena. En ese contexto, se hace necesario abrir nuevos espacios de fortalecimiento del patrimonio cultural, que se logrará no solamente combatiendo el analfabetismo y el rezago social de las comunidades indígenas, sino de buscar alternativas sustentables y autogestivas en el aspecto formativo para asegurar la reproducción de nuevos ciudadanos indígenas bilingües. Críticos, orgullosos de su pasado histórico y constructivos, en el marco de una educación indígena. (leepo 1998, p. 1)

En suma, las experiencias de formación se están transformando en dos sentidos: uno, tienden a una mayor especialización y, dos, ensayan 
modalidades de intervención en la práctica docente, a fin de introducir los cambios en el terreno del aula. Las propuestas se orientan a la educación de niños arraigados en su medio, con las competencias necesarias para vivir en él y desarrollarlo con los elementos que les ofrece su herencia sociocultural y los aportes de la ciencia. "Desde esta perspectiva se articulan los conocimientos indígenas con los científicos, se reflexiona sobre los valores indígenas y se recogen las estrategias y contextos de aprendizaje propios de los pueblos que se complementan con los aportes de la psicopedagogía" (ISP Loreto 1997, p. 20).

\title{
Modelos y diseños académicos de la educación indígena
}

\begin{abstract}
Las deficiencias de la educación en las zonas indígenas se deben no sólo a la irregularidad y las limitaciones de los servicios, sino también a un enfoque pedagógico y cultural inadecuado, que se origina en el intento de reproducir, con adaptaciones marginales y bajo condiciones precarias, el esquema genérico de la escuela urbana como forma básica del servicio educativo. En suma, la política del gobierno federal, concertada con las autoridades estatales, tendrá dos orientaciones centrales: consolidar y extender los programas compensatorios para las escuelas de zonas indígenas (...) y flexibilizar los contenidos curriculares, las formas organizativas y normas académicas de la escuela. En síntesis, se trata de avanzar hacia nuevos modelos de educación indígena, con rasgos propios en los aspectos académicos y en las formas de operación. Los modelos habrán de incorporar como criterio ordenador la gran diversidad de modos de vida y cultura de los pueblos indígenas. Este hecho exige una gran adaptabilidad de la acción educativa a las condiciones y expectativas de las poblaciones en el nivel de comunidad y microrregión. La Secretaría de Educación Pública emitirá las normas para la formulación de las adaptaciones antes mencionadas. Evidentemente, debe existir igual flexibilidad respecto a horarios, calendarios y otras reglas organizativas del servicio escolar. (SEP 1996, pp. 76-77)
\end{abstract}

La estrategia que se propone al respecto de esa población consiste en promover durante los primeros grados el aprendizaje inicial de la lectura y la escritura en la lengua materna, e iniciar, cuando los avances logrados en los primeros grados lo permitan, la enseñanza oral y 
escrita del español como segunda lengua. En los grados educativos más avanzados se consolidará el uso del español, pero se propiciará también la expresión en lengua materna. La aplicación de esa orientación demandará elevar la capacidad lingüística de los educadores, en correspondencia con las poblaciones que éstos atienden (p. 79).

Una importante experiencia innovadora es el Bachillerato Integral Comunitario Ajuujk Polivalente (Bicap), que postula una concepción del ser humano que tiene como don y como tarea la realización de su ser como persona. Esa propuesta surgida en Santa María Tlahuitoltepec, región Mixe Alta de Oaxaca, concibe la educación como el conjunto de decisiones y acciones que conforman la historia personal, como un quehacer cotidiano intencionado que realiza a cada cual. La educación es un derecho inalienable del ser humano y, a la vez, el proceso que lo construye humanamente como persona (Yinet 1998, p. 11).

Según la propuesta, la educación actual en Santa María Tlahuitoltepec inhibe el desarrollo normal cognoscitivo, psicomotor, afectivo y social del niño, por el uso de una lengua ajena de su hogar y espacio vital; eso le crea un conflicto psicológico, el cual se manifiesta en una personalidad insegura, aunada a una indefinición cultural. Se inhibe la creatividad, la motivación al estudio y la capacidad de diálogo, por la dependencia excesiva hacia el educador - esto se da en todos los niveles educativos. La teoría está desligada de la práctica, y la escuela es una élite dentro de la comunidad, cuando podría ser parte de ella.

En la articulación actual del sistema escolarizado, la educación media superior está marcando decisiones sociales que acentúan los niveles de injusticia social. Si, por una parte, el país soporta ya una sobrecarga de profesionistas que nuestra realidad no requiere, y condena a sus egresados al desempleo, por otra, existe una demanda cada vez mayor de la población que desea tener acceso a un nivel educativo más avanzado. Existe también una profunda necesidad de estudios y profesiones vinculadas a un desarrollo económico, cultural y social, que actúe particularmente en las zonas rurales e indígenas. Por eso, la propuesta Bicap asume como propósitos generales: 1) la formación de personas prácticas, científicas, técnicas, creativas e inventivas, con capacidad de teorizar la vida y la realidad de su entorno inmediato para entender su existencia individual y social en el contexto nacional e internacional; 2) el desarrollo de habilidades y actitudes propositivas para el mejoramiento de las actividades socioproductivas sustentables en la comunidad y sociedad en general, por intermedio del núcleo familiar, y 3) la interiorización de responsa- 
bilidad y compromiso con la identidad cultural, valorizando el idioma, las expresiones artísticas, conocimientos y prácticas comunitarias trascendentes que fundamentan la construcción del desarrollo holístico prospectivo (Yinet 1998, pp. 12-13).

Experiencias como éstas replantean la función de la educación en los entornos indígenas interculturales, aspiran a la integralidad y reconstitución de las relaciones escuela-comunidad, al reconocimiento de las biodiversidades ecológicas y se sustentan en algún modelo de investigación-acción-participación. Sin embargo, hay que admitir que es el nivel menos elaborado dentro de las dimensiones del desarrollo educativo que comentamos. Prácticamente, dominan las iniciativas puntuales tendientes a elevar la calidad del clima sociopedagógico del aula y a fomentar el uso oral y escrito de la lengua indígena en el contexto escolar.

La dimensión decisiva: Las prácticas específicas de la educación indígena

En las escuelas indígenas se expresan desigualmente las innovaciones propiciadas desde las instituciones creadoras de política educativa y desde el sistema educacional. Por una parte, se ha ganado bastante terreno en infraestructura y edificios escolares, en la producción de textos gratuitos en lengua indígena materna y en eventos de actualización-capacitación. En torno al diseño curricular y la oferta formadora en educación bilingüe aparecen más iniciativas. En torno a otros factores acontecen cada vez más avances y experiencias: el diseño curricular y la profesionalización en educación intercultural bilingüe. Sin embargo, todavía existe poco progreso en la constitución formativa y comprensiva de la enseñanza-aprendizaje en el aula indígena, en la inmensa mayoría de los casos. Y resulta verdaderamente un misterio estimar cuándo se lograrán estos objetivos, que podemos definir como de calidad y pertinencia educativa.

Así, el proyecto educativo intercultural bilingüe que se observa en las prácticas curriculares y programáticas responde de manera parcial a las propuestas de la política educativa oficial, porque en los contextos comunitarios cobran más relevancia los contactos interétnicos y las necesidades que de ahí emanan: igualación social, valorización, acceso al trabajo. 
Algunas reflexiones finales

La educación intercultural bilingüe, a pesar de no ser una propuesta surgida originalmente desde los pueblos indígenas, ha abierto espacios para crear una nueva relación entre el Estado mexicano, organizaciones indígenas y otros sectores sociales. Y su legitimidad obedece tanto a la forma de encarar o acordar un cambio como al propio contenido de ese cambio. Pero el desarrollo de la educación en regiones indígenas mexicanas seguirá impedido mientras no haya congruencia e integración en las diversas dimensiones mencionadas. Dicho de otro modo, la modernización o mayor eficiencia de una de esas dimensiones no garantiza el cambio hacia la calidad o pertinencia educativa.

A la fecha, los niveles con mayor dinámica y crecimiento son el de la política y proyectos educativos y el de sistema educacional. El nivel de los modelos académicos de formación, de hecho, se reduce a propuestas sobre la enseñanza y aprendizaje de la lectura y escritura de los alumnos indígenas. La práctica docente se realiza todavía sobre bases idiosincráticas y reglas locales, poco permeables por la ideología oficial de la modernización educativa.

En México sigue siendo una gran necesidad el esclarecer el entorno contemporáneo de la diversidad lingüística y cultural, situación que ha vuelto a poner en el tapete de la teoría del lenguaje la discusión sobre la utilidad o alcance de las descripciones lingüísticas, estudios censales y diversos tipos de intervenciones sobre la estandarización de los idiomas.

Las comunidades y escuelas indígenas, desde un horizonte de ruralidad, con sus lógicos particularismos, interpretan y operacionalizan las nuevas modalidades de la educación indígena mexicana. En todo caso, muestran que está en curso un proceso de evaluación de la escuela pública indígena y que emerge un componente democrático y participativo, a través del cual la comunidad indígena respectiva profundiza la descentralización (en abierta oposición al sistema centralizado) y explora un mayor margen de diversificación en el trabajo escolar.

La información científica que emana de las investigaciones y evaluaciones es una fuente muy confiable para replantear la construcción curricular y la concepción pedagógica de la escolarización. En efecto, si examinamos el desarrollo educativo desde la perspectiva de los procesos 
escolares específicos, se hacen notables algunas incompatibilidades entre los postulados de la política educativa y la escolarización, en virtud de concepciones sobre la enseñanza, el conocimiento y el aprendizaje en las aulas indígenas, que responden a principios teóricos y didácticos claramente discrepantes de los formulados formalmente en las políticas y proyectos educativos. Dicho de otro modo, formas actuales de la metodología didáctica en las escuelas indígenas han independizado, o simplemente separado, el espacio y la orientación de la enseñanza-aprendizaje de actitudes y competencias básicas escolares de la práctica social de las competencias básicas en la vida cotidiana de los alumnos. Es decir, aunque se desarrollan extensos procesos escolares de enseñanza y aprendizaje de la lectura, por ejemplo, los alumnos no se forman ni como lectores de textos escolares ni sociales. Análogamente, se enseña la escritura, pero los alumnos no producen textos escritos, no desarrollan la capacidad de expresarse en la lengua escrita cotidianamente, y así con todas las mutaciones señaladas.

A modo de hipótesis articuladora de las cuatro dimensiones del desarrollo educativo, planteo que la transformación o enriquecimiento más importante de la educación en regiones indígenas provendrá principalmente del nivel de las realidades y prácticas escolares, en la medida que se democraticen la gestión y los recursos de las políticas de educación pública, mediante formas de control comunitario, y en la medida que se hagan más eficientes y pertinentes los procesos de enseñanza y aprendizaje. El sistema educacional sólo reflejará las condiciones políticas imperantes. El nivel de los modelos académicos o de formación permanecerá invisible, hasta que se restablezcan las condiciones profesionales y las conexiones con la investigación de la educación indígena.

Quizás uno de los desafíos será posibilitar auténticamente la intervención del profesorado y de las comunidades indomexicanas, lo cual no es posible en la actualidad por razones de formación insuficiente, escasa información y relaciones no democráticas.

\section{Public policy and school indigenous education}

ABSTRACT: The education of the indigenous students, in any of its modalities, however, hasn't got the status of the main issue in the present Mexican society, though it has obtained great presence in the political activity and in the cultural scenarios. Though political debate 
is incapable to connect the educative development with political and economic reforms, that today divide deeply the Mexican nation however, they don't visualize the structural changes corresponding to a State that coherently assumes its ethnic diversity. Since the educational system, the demands about bilingual education and cultural multiplicity have shown much less influence than the modernization doctrine from basic education and less interesting that the struggle for the hegemony from such pedagogical paradigms in the high rankings of the Educational Institutions. Summarizing, the indo-mexican schools and communities should regain their education, at the same time that they need participation and decision capacity to tackle the government institutions.

Bibliografia

BRAVO AHUJA, Gloria. La enseñanza del español a los indígenas mexicanos. México: El Colegio de México, 1977.

CÁRDENAS, Víctor H. "Discurso de inauguración". II Congreso latinoamericano de educación intercultural bilingüe: Las lenguas indígenas dentro y fuera de la escuela. Santa Cruz de la Sierra, Bolivia, 1997.

Compromisos y propuestas conjuntas de los gobiernos del Estado y Federal y el EZLN, 16 de febrero de 1996.

CORONADO, Gabriela et al. "Educación y bilingüismo en el Valle del Mezquital”. Cuadernos de la Casa Chata. México: Ciesas, 1980.

DÍAZ-COUDER, Ernesto. "Diversidad sociocultural y educación en México". Seminário Educación y Cultura: La reflexión actual en México. México, 1992.

GARMENDIA, Jesús e SAINZ, Matilde. "Formación de docentes bilingües. El caso del país vasco". III Simposio internacional sobre autonomia de la Costa Atlántica de Nicaragua. Managua, 12-15/10/1997.

GOSSEN, Gary. Los chamulas bajo el mundo del sol. México: INI \& Conaculta, 1979.

GUMPERZ, John. "El significado de la diversidad lingüística y cultural en los contextos postmodernos". In: MUÑOZ, H. e LEWIN, P. (orgs.). EI significado de la diversidad lingüística y cultural. Investigaciones lingüísticas, vol. 2. México: UAM-Iztapalapa, 1996, pp. 33-48. 
HAMERS, Josiane e DA SILVEIRA, Ivonne. "Vitalité ethnolinguistique, développement multilingue et langue d'enseignement en Afrique". Des langues et des villes. Paris: Didier Erudition, 1993, pp. 339-346.

INSTITUTO ESTATAL DE EDUCACIÓN PÚBLICA DEL ESTADO DE OAXACA (IEEPO). Proyecto de escuela normal bilingüe de Oaxaca. Ms., 1998.

INSTITUTO SUPERIOR PEDAGÓGICO LORETO. "Lineamientos curriculares. Formación Magisterial en la especialidad de educación primaria intercultural bilingüe". Iquitos, Perú: Programa de formación de maestros bilingües de la Amazonía Peruana \& Asociación Interétnica de Desarrollo de la Selva Peruana, 1997.

LASTRA, Yolanda; HAMEL, Rainer e MUÑOZ, Héctor. Sociolingüística latinoamericana. México: Unam, Instituto de Investigaciones Antropológicas, 1989

LATAPÍ, Pablo. "El programa educativo para 1997". Revista Proceso 1061, 1997.

LE BOT, Yvon. Subcomandante Marcos. El sueño zapatista. México: Plaza \& Janés, 1997.

LESOURNE, Jacques. Educación y sociedad. Los desafíos del año 2000. Barcelona: Gedisa, 1993.

MEC. Referencial curricular nacional para a escola indígena (RCNE/indígena). Parte I "Para começo de conversa". Brasília: MEC/Coordenação Geral de Apoio às Escolas Indígenas, 1997.

MENA, Patricia; MUÑOZ, Héctor \& RUIZ, Arturo. Identidade, lenguaje y enseñanza: Impactos actuales de la escuela indígena de Oaxaca. México, Oaxaca, Universidad Pedagógica Nacional-Oaxaca, 1998 (en prensa).

MINISTERIO DE EDUCACIÓN DE CHILE (Coordinación Programa Educación Intercultural Bilingüe). Orientaciones generales referentes al programa de educación intercultural bilingüe. Chile: División de Educación General, 1996.

MODIANO, N. La educación indígena en los Altos de Chiapas. México: INI \& SEP, 1974.

MUÑOZ, Héctor et al. Una experiencia de alfabetización bilingüe en comunidades mazahuas del Estado de México. México: DIE-Cinvestav \& Conacyt, 1989. 
MUÑOZ, H. e LEWIN, P. (orgs.). El significado de la diversidad lingüística y cultural. Investigaciones Lingüísticas, vol 2. México: UAMIztapalapa 7 Centro INAH - Oaxaca, 1996.

PODER EJECUTIVO FEDERAL. Programa de desarrollo educativo 19952000. México: Secretaría de Educación Pública, 1996.

PODESTÁ, Rossana. "La función de la escuela en la cultura oral nahuatlata (Análisis de dos casos educativos contrastantes de vitalidad y desplazamiento lingüístico)". Tesis de maestría. México: Depto. de Investigaciones Educativas, Cinvestav, 1993.

PODESTÁ, R.; CHILACE, C. e MARTÍNEZ, E. "La perspectiva del alumno náhuatl y otomí en la refuncionalización del discurso oral y escrito". In: MUÑOZ, H. e LEWIN, P. (orgs.), pp. 269-278.

SALINAS, Gisela et al. Evaluación y seguimento de las licenciaturas en educación preescolar y en educación primaria para el medio indígena (LEP y LEPMI 90). Segunda etapa. México: Universidad Pedagógica Nacional, 1997.

SCANLON, Patricia e LEZAMA, Juan (orgs.). Educación bilingüe en México. México: Dirección Gral. de Educación Indígena, 1988.

SECRETARÍA DE EDUCACIÓN PÚBLICA-PODER EJECUTIVO FEDERAL. Programa de desarrollo educativo 1995-2000. México: Secretaría de Educación Pública, 1996.

SIDLER, Rolf. La representación de las culturas indígenas mexicanas en los libros de textos gratuitos a partir de la influencia del movimiento multicultural. Universidad de Zurich, ms., 1995.

SZEKELY, Gabriel. "Llamado urgente a la educación". El Universal (notas editoriales). México, DF, 12/2/1997.

ZIMMERMANN, Klaus. Lenguas en contacto en Hispanoamérica. Frankfurt: Biblioteca Ibero-Americana, Vervuet Verlag, 1995.

YINET. Yiwejin kajï jawyee njïnptua'ant, ํํ⒈ México, Oaxaca, junho 1998. 